\title{
BRIEF COMMUNICATION OPEN Drinking water bacterial communities exhibit specific and selective necrotrophic growth
}

\author{
Ioanna Chatzigiannidou ${ }^{1}$, Ruben Props $^{1}$ and Nico Boon ${ }^{1}$
}

Physicochemical water disinfection methods result in the reduction of bacterial concentrations by orders of magnitude, but not in the total elimination of the bacterial community. As such, the dead bacterial biomass may act as a carbon and nutrient source for the survivor populations. The ability of bacterial strains to grow on dead bacterial cells has been described before as necrotrophy. We investigated the impact of killed bacterial biomass of two different bacterial strains on the growth potential of natural drinking water microbial communities. Many indigenous bacterial taxa could grow on dead biomass, with the total bacterial concentration increasing from $10^{4}$ to $10^{8}$ cells $/ \mathrm{ml}$. Necrotrophic growth was specific ( 43 enriched taxa) and selective (i.e. enriched taxa were dependent on the type of dead biomass). The potential of natural water communities to grow necrotrophically has remained underexplored. Nevertheless the phenomenon can have a big impact in water quality and deserves more attention.

npj Clean Water (2018)1:22; doi:10.1038/s41545-018-0023-9

\section{INTRODUCTION}

Water disinfection is an essential procedure to decrease the microbial load in water sources and make them safe for consumption. Disinfection can be attained chemically (e.g. chlorination) or physically (e.g. heat, UV). Although the procedure is able to decrease the microbial load, it cannot eliminate all microorganisms. At the same time, the dead bacterial cells remain in the water resulting in an increase of available nutrients (e.g. nitrogen, carbon and phosphorus), which are important elements for bacterial growth. Current research on disinfection does not take into account the impact of this biomass excess on the survivor populations and the water quality. However, certain bacterial strains can use dead bacterial cells as a nutrient source, ${ }^{1,2}$ a phenomenon referred to as necrotrophy or necrotrophic growth. ${ }^{1}$ Necrotrophic growth has been studied in axenic cultures, but it has not been described before in a mixed microbial community.

In this study, we tested how the natural microbial community of a drinking water source responds to an excess dead bacterial biomass $\left(10^{7}\right.$ cells $\left./ \mathrm{ml}\right)$. Evian bottled water was used as a benchmark water source due to its stable microbial community. Escherichia coli $\left(\mathrm{Gram}^{-}\right)$and Lactobacillus brevis $\left(\mathrm{Gram}^{+}\right)$were added to the water to test if the type of the dead biomass would drive the structure of the microbial community. Dead biomass was generated either by autoclavation (physical disinfection) or $5 \%$ chloroform (fumigation-chemical disinfection) to examine whether the killing method influences the response of the natural community. Yeast extract (YE) $(0.5 \mathrm{~g} / \mathrm{l})$ supplementation was used as benchmark control. The concentration of the living and dead biomass was monitored with flow cytometry (FC) in 24 intervals over 7 days. 16S rRNA gene amplicon sequencing was used to determine the bacterial community composition.

\section{RESULTS AND DISCUSSION}

The microbial community as a whole could grow necrotrophically on the different types of dead biomass (Fig. 1a). The cell concentration increased from $10^{4}$ cells $/ \mathrm{ml}$ to $10^{7}-10^{8}$ cells $/ \mathrm{ml}$. Samples that received dead biomass reached higher cell concentrations than the positive control, although the added carbon load was less (chemical oxygen demand was $8.5-22.8 \mathrm{mg} / \mathrm{l}$ for dead biomass samples and $675 \mathrm{mg} / \mathrm{l}$ for YE) (Fig. 1b). The $E$. coli-supplemented samples reached significantly ( $p$-value $<0.01)$ higher cell concentrations than the ones supplemented with $L$. brevis (Fig. 1a). The phenomenon that dead $\mathrm{Gram}^{+}$cells exhibited lower capacity to stimulate growth was previously observed in axenic cultures, ${ }^{1,2}$ potentially due to their robust cell wall that increases the difficulty for other bacteria to access their nutrients. ${ }^{1}$ An alternative explanation could be that an aquatic water community, consisting mostly of $\mathrm{Gram}^{-}$strains, has adapted more to the consumption of $\mathrm{Gram}^{-}$dead biomass.

$16 \mathrm{~S}$ rRNA gene amplicon sequencing revealed that there were compositional differences at the genus level between treated and non-treated samples. The most abundant phyla, identified in all samples, were Proteobacteria, followed by Bacteroides, Actinobacteria, and Firmicutes, as it has shown before for bottled water microbial communities. ${ }^{3,4}$ Nevertheless, there were differences at the genus level between treated and non-treated samples. The most abundant taxa identified in the control samples were unclassified Burkholderiales (Fig. 2). The YE-treated samples revealed the most similar bacterial composition among the biological replicates, with Hydrogenophaga being the most abundant genus (Fig. 2). The most abundant genera in the necrotrophic grown samples were Brevudimonas, Stenotrophomonas, Pseudomonas and Rhizobium (Fig. 2). However, large variability was observed in relative abundances between treatments as well as biological replicates of the same treatment (Fig. 2). The variability within biological replicates could not be

${ }^{1}$ Center for Microbial Ecology and Technology (CMET), Ghent University, Gent, Belgium

Correspondence: Nico Boon (Nico.Boon@UGent.be)

Received: 22 February 2018 Revised: 20 September 2018 Accepted: 24 September 2018

Published online: 21 November 2018 

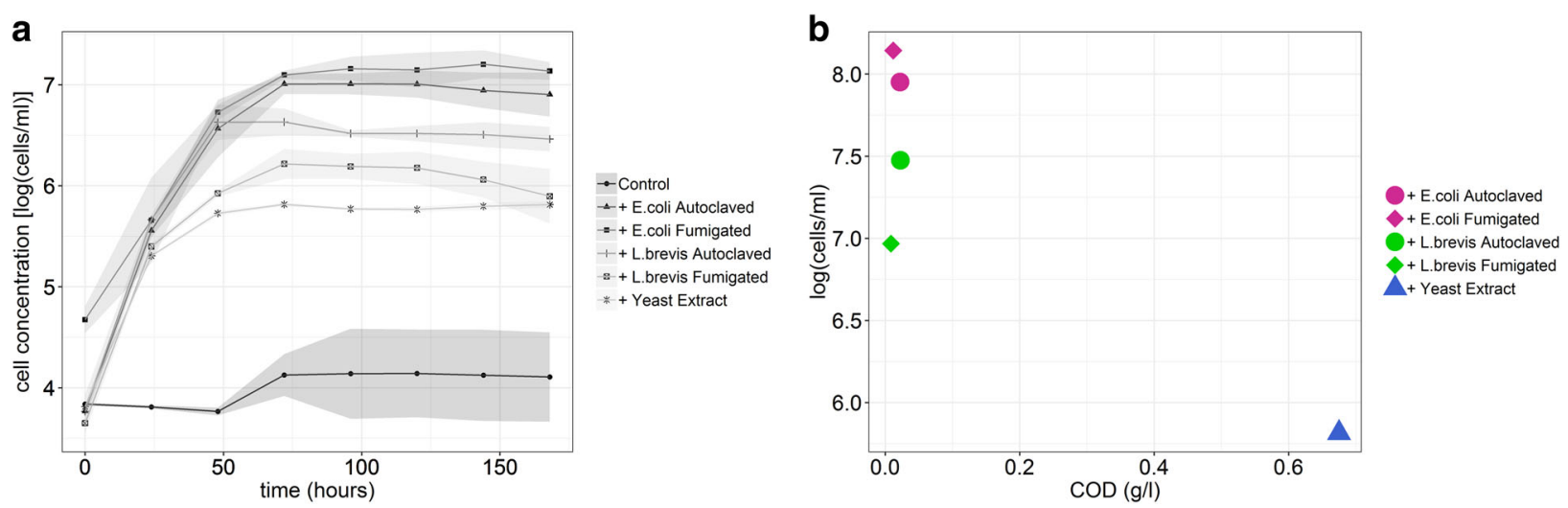

Fig. 1 a Bacterial growth: Live cell concentrations was measured with flow cytometry and SYBR Green I/Propidium lodide staining in 24 intervals for a period of 7 days. The displayed concentration represents the average of four biological replicates and the ribbon the standard deviation. b Final cell concentration of natural drinking water bacterial community after necrotrophic growth compared to the added COD concentration of the different treatments
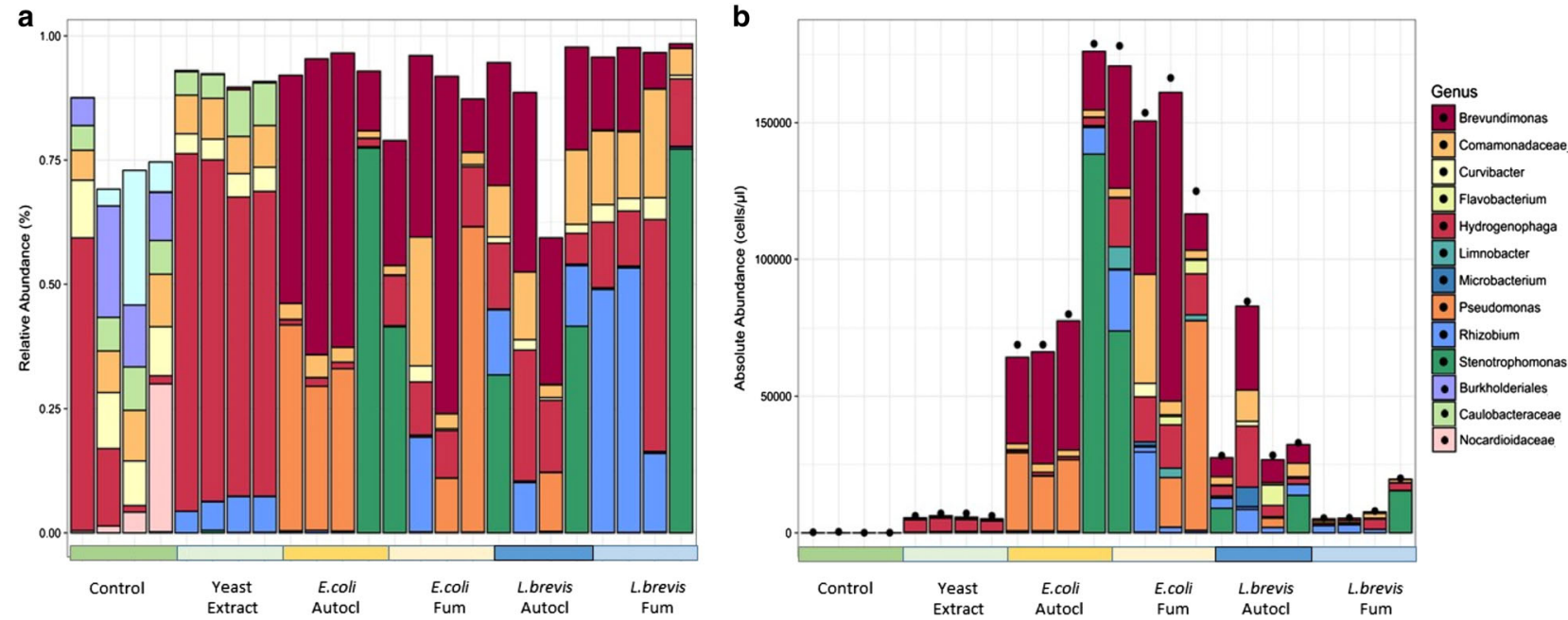

Fig. 2 a Relative abundances of the 10 most abundant genera. b Absolute abundances of 10 most abundant genera. Dots depict the total cell density per sample

explained by differences in absolute abundances, in all cases (Fig. $2 b)$. Thus necrotrophic growth may possibly be subject to stochasticity and the initial bacterial composition.

To examine the effect of necrotrophy on specific taxa, the differentially abundant taxa between the non-treated samples and each treatment were identified (Fig. 3a). Forty-three taxa were enriched in at least one of the treatments $\left(p_{\mathrm{adj}}<0.01\right)$. Moreover, the specific effect of the supplemented biomass was investigated by identifying the differentially enriched taxa between the two strains that were used as supplements or the two methods of generating dead biomass. Twenty-three taxa were differentially enriched in E. coli- versus L. brevis-supplemented samples (Fig. 3b), whereas there were no significant $\left(p_{\text {adj }}>0.01\right)$ differences between autoclaved or fumigated supplements.

The changes on the bacterial community due to necrotrophy were also depicted in the alpha diversity of the samples. Alpha diversity is the within-sample diversity, with taxonomic to refer to the observed diversity based on the amplicon sequencing data, while phenotypic diversity refers to the changes of the phenotypic profile (fingerprint) of the community, measured by FC. ${ }^{5}$ Lower taxonomic and phenotypic alpha diversity were observed for all treated samples compared to the negative control (Fig. 3c, d). The above indicates a selective effect with specific taxa/phenotypes benefiting more by the available dead biomass.

This study revealed that the natural bacterial community of drinking water exhibited necrotrophic growth. However, certain bacterial taxa exhibited better efficacy at necrotrophy, as indicated by the differential abundance of the enriched taxa and the lower diversity of the treated samples. Moreover, the differentially enriched taxa between $E$. coli- and L. brevis-treated samples revealed that the type of biomass affected the behaviour of the community.

Overall, the increased cell concentration and the altered community composition has the potential to greatly influence the water quality. For example, the decreased diversity observed during necrotrophy could lead to decreased invasion resistance. ${ }^{6,7}$ While the short amplicon sequences prevented the identification of pathogenic strains that were specifically benefiting from necrotrophy, isolation with selective medium, or targeted PCR/ qPCR could be used in the future to identify possible pathogens. ${ }^{2}$ Nevertheless, we argue that an excess of dead bacterial biomass can have a substantial impact on oligotrophic microbial dynamics and thus further research is needed to determine the impact of necrotrophic growth during water disinfection. 
a
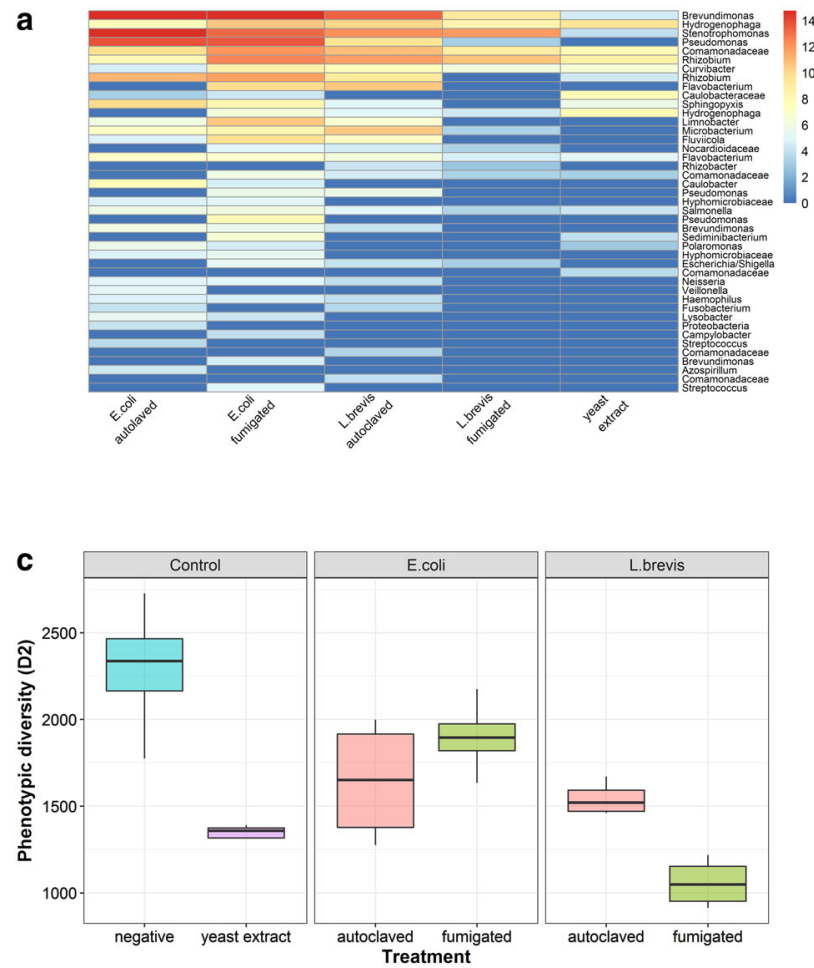

Treatment 追 autoclaved 白 fumigated 追 negative 追 yeast extract
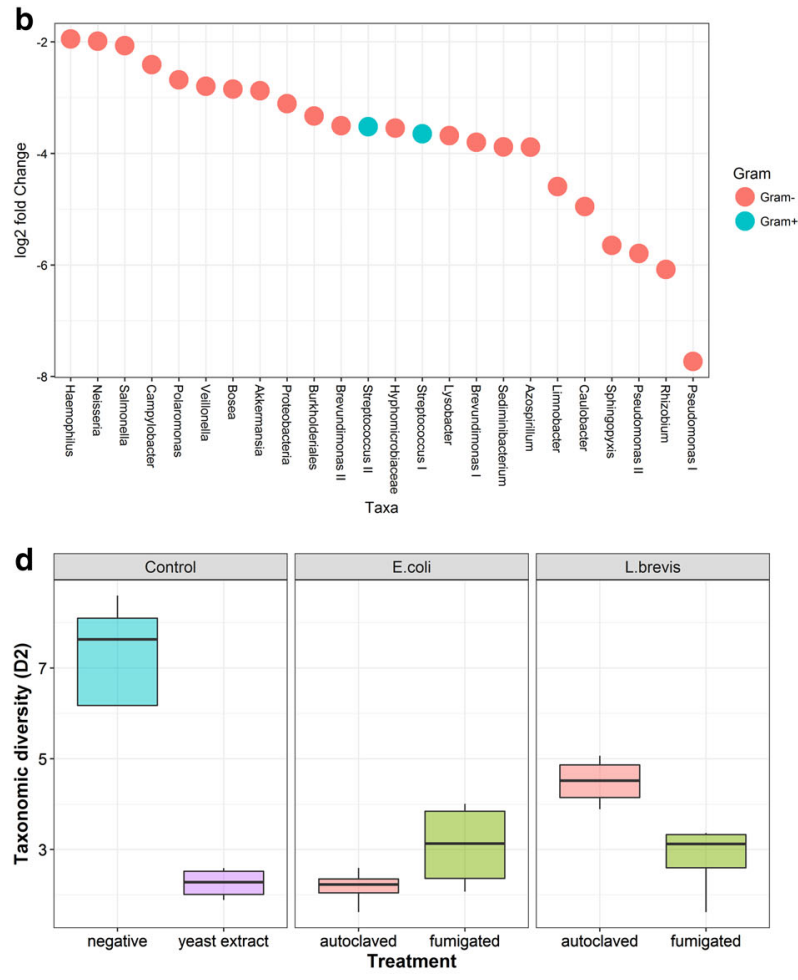

Treatment 官 autoclaved 追 fumigated 追 negative 追 yeast extract

Fig. 3 a Differentially enriched taxa in treated samples compared to non-treated control samples. Differentially abundant taxa were calculated using the package DESeq2 in R ( $p$-value $<0.01)$. b Differentially enriched taxa in $E$. coli-treated samples compared to $L$. brevis-treated samples. Negative values indicated that the taxa were more abundant in $E$. coli-treated samples. Phenotypic Diversity $\left(D_{2}\right)$ c or taxonomic diversity $\left(D_{2}\right)$ d calculated from flow cytometric fingerprinting or $16 \mathrm{~S}$ rRNA amplicon sequencing data accordingly for the last time point $(t=7$ days) using the Hill Number framework (order 2). Control (non-treated) samples exhibited the highest diversity in both cases

\section{MATERIAL AND METHODS}

Dead biomass production

Escherichia coli (DSM 2840) and Lactobacillus brevis (LMG 18022) were grown overnight in LB (Sigma Aldrich, Belgium) and MRS broth (Oxoid, UK), respectively. The cultures were resuspended in PBS (phosphate-buffered saline tablets; Sigma Aldrich, Belgium) and split in two. One part of the solution was autoclaved (following standard autoclavation cycle $121{ }^{\circ} \mathrm{C}$ for $15 \mathrm{~min}$ ) and the other part was fumigated by addition of $5 \%$ chloroform (Honeywell Fluka, Sigma Aldrich, Belgium) for $1 \mathrm{~h}$. The samples treated with chloroform were subsequently washed twice with PBS to remove the remaining chemical. Flow cytometry was used to calculate the cell concentration in each sample.

Fifty microliters of each condition were plated in LB (Sigma Aldrich, Belgium) and MRS agar plates (Oxoid, UK), respectively, and incubated in $28^{\circ} \mathrm{C}$ for $48 \mathrm{~h}$ to verify that there were no survivals. There were no colonies observed in any of the agar plates.

The COD (chemical oxygen demand) was analysed with Nanocolor ${ }^{\circledR}$ kits (028; Macherey-Nagel).

\section{Experiment}

Evian bottled water (Evian, France) was used as the drinking water source. Equal concentrations $\left(10^{7}\right.$ cells $/ \mathrm{ml}$, as calculated by flow cytometry) of autoclaved E. coli, fumigated $E$. coli, autoclaved $L$. brevis and fumigated $L$. brevis were added in different bottles of drinking water. This concentration of dead cells corresponded to a COD of $22 \mathrm{mg} / \mathrm{l}$ for autoclaved E.coli, $12 \mathrm{mg} / \mathrm{l}$ for fumigated E.coli, $22.5 \mathrm{mg} / \mathrm{l}$ for autoclaved $L$. brevis and $8.5 \mathrm{mg} / \mathrm{l}$ for fumigated $L$. brevis. Addition of $0.5 \mathrm{~g} / \mathrm{l}$ ( $675 \mathrm{mg} / \mathrm{l}$ COD) yeast extract (Oxoid, UK) was used for the positive control. Every treatment was done in quadruplicates and all the samples were maintained in $28^{\circ} \mathrm{C}$ for a period of 7 days.

\section{Flow cytometry}

In $24 \mathrm{~h}$ interval, samples were taken from every bottle for flow cytometric measurements. The samples were diluted in sterile PBS and stained with the nucleic acid stain SYBR Green I and Propidium lodide that stains permeabilized cells. SYBR Green I $(10,000 x$ concentrate in dimethyl sulfoxide (DMSO); Invitrogen) was diluted 100 times in $0.22-\mu \mathrm{m}$-filtered DMSO (IC Millex, Merck, USA) and Propidium lodide (20 mM in DMSO; Invitrogen, USA) was diluted 50 times. Following, they were incubated in $37^{\circ} \mathrm{C}$ for $13 \mathrm{~min}$. Measurements were performed with a FACSVerse cytometer (BD Biosciences, Belgium). The performance of the instrument was monitored daily and the instrument was calibrated with the CS\&T calibration beads (BD Biosciences, Belgium). The blue laser $(488 \mathrm{~nm}$ ) was used for the excitation of the stains. A minimum of 10,000 cells per sample were measured to allow accurate quantification.

The data of each sample were denoised from (in)organic noise by a filtering approach using the flowCore package (v1.38.1) in $\mathrm{R}$ (v3.3.2). The bacterial cell population was extracted by a gate applied on the primary fluorescence emission channels (green $(530 / 30 \mathrm{~nm})$ and red $(>670 \mathrm{~nm}))$.

\section{DNA extraction and amplicon sequencing}

The remaining water volume was filtered through $0.2 \mu \mathrm{m}$ filter and the filters were stored in $-20^{\circ} \mathrm{C}$. DNA was extracted with the PowerWater Kit (MO BIO, Belgium). The quality of the DNA extracts was validated by means of agarose gel electrophoresis and via PCR. The quality of the PCR product was determined with agarose 
gel electrophoresis to ensure that no inhibition of the PCR took place.

The DNA extracts were sent to LGC Genomics (GmbH, Berlin, Germany) for $16 \mathrm{~S}$ rRNA amplicon sequencing. High-throughput amplicon sequencing of the V3-V4 hypervariable region ${ }^{8}$ (using the 341F CCTACGGGNGGCWGCAG and 785Rmod GACTACHVGGGTATCTAAKCC primers) was performed with the Illumina MiSeq platform, according to the manufacturer's guidelines. Analysis of the amplicon data was performed with the software package MOTHUR (v.1.33.3). ${ }^{9}$ Contigs were generated by merging the forward and reverse reads and they were further aligned to the SILVA v123 database. Sequences that did not correspond to the V3-V4 region as well as sequences with ambiguous bases or more than 12 homopolymers were removed. The aligned sequences were filtered and dereplicated. UCHIME was used to removed chimeras $^{10}$ and the sequences were clustered in OTUs with $97 \%$ similarity with the cluster.split command (average neighbour algorithm). OTUs were subsequently classified using the SILVA v123 database.

\section{Data analysis}

All statistical analysis was performed in R (v3.3.2). Welch twosample $t$-test, 'stats' package, was used to test for significant difference between cell concentrations of samples fed with $E$. coli or L. brevis. The phenotypic fingerprint based on flow cytometry data was calculated using the PhenoFlow package (v1.0) in R (v3.3.2). ${ }^{5}$ The same package was used to calculate the phenotypic alpha diversity based on the Hill Number framework.

The OTU contingency table was imported in R. OTUs with no more than one read in every sample (singletons) were removed. ${ }^{11}$ The pre-processed reads were normalized over the cell concentrations acquired with flow cytometry to receive the absolute OTU abundances. $^{12}$ The graphs representing the 10 most relative or absolute abundant genera were generated using the phyloseq package $^{13}$ in R (v3.3.2). Finally the differentially enriched samples were calculated using the DESeq2 package (v1.16.1) after the data underwent prevalence filtering (prevalence >0.05). ${ }^{11}$ The adjusted $p$-value $\left(p_{\text {adj }}\right)$ was calculated based on Benjamini and Hochberg method and $p_{\text {adj }}<0.01$ was considered significant. The taxonomic alpha diversity was also calculated according to the Hill Number framework (order 2).

\section{DATA AVAILABILITY}

Flow cytometry data (fcs format) are available on the FlowRepository archive under repository ID FR-FCM-ZYG7. Sequences are available on the NCBI Sequence Read Archive (SRA) under accession number SRP130062.

\section{ACKNOWLEDGEMENTS}

This work was supported by the IMPROVED project, subvented by The interreg $V$ "Vlaanderen-Nederland" program, a programme for transregional collaboration with financial support from the European Regional Development Fund. More info: www. grensregio.eu (in Dutch).

\section{AUTHOR CONTRIBUTIONS}

N.B. and I.C. designed the study. I.C. performed the experiments and wrote the manuscript. I.C. and R.P. analysed the data. All authors reviewed and approved the manuscript.

\section{ADDITIONAL INFORMATION}

Competing interests: The authors declare no competing interests.

Publisher's note: Springer Nature remains neutral with regard to jurisdictional claims in published maps and institutional affiliations.

\section{REFERENCES}

1. Temmerman, R., Vervaeren, H., Noseda, B., Boon, N. \& Verstraete, W. Necrotrophic growth of Legionella pneumophila. Appl. Environ. Microbiol. 72, 4323-4328 (2006).

2. Rodriguez Herrero, E. et al. Necrotrophic growth of periodontopathogens is a novel virulence factor in oral biofilms. Sci. Rep. 7, 1107 (2017).

3. França, L., Lopéz-lopéz, A., Rosselló-móra, R. \& Costa, M. S. Microbial diversity and dynamics of a groundwater and a still bottled natural mineral water. Environ. Microbiol. 17, 577-593 (2015).

4. Loy, A., Beisker, W. \& Meier, H. Diversity of bacteria growing in natural mineral water after bottling. Appl. Environ. Microbiol. 71, 3624-3632 (2005).

5. Props, R., Monsieurs, P., Mysara, M., Clement, L. \& Boon, N. Measuring the biodiversity of microbial communities by flow cytometry. Methods Ecol. Evol. 7, 1376-1385 (2016).

6. Kennedy, T. A. et al. Biodiversity as a barrier to ecological invasion. Nature 417, 636-638 (2002).

7. De Roy, K. et al. Environmental conditions and community evenness determine the outcome of biological invasion. Nat. Commun. 4, 1383-1385 (2013).

8. Klindworth, A. et al. Evaluation of general $16 \mathrm{~S}$ ribosomal RNA gene PCR primers for classical and next-generation sequencing-based diversity studies. Nucleic Acids Res. 41, 1-11 (2013).

9. Schloss, P. D. et al. Introducing mothur: open-source, platform-independent, community-supported software for describing and comparing microbial communities. Appl. Environ. Microbiol. 75, 7537-7541 (2009).

10. Edgar, R. C., Haas, B. J., Clemente, J. C., Quince, C. \& Knight, R. UCHIME improves sensitivity and speed of chimera detection. Bioinformatics 27, 2194-2200 (2011).

11. McMurdie, P. J. \& Holmes, S. Waste not, want not: why rarefying microbiome data is inadmissible. PLoS Comput. Biol. 10, e1003531 (2014).

12. Props, R. et al. Absolute quantification of microbial taxon abundances. ISME J. Adv. online Publ. 1-4, https://doi.org/10.1038/ismej.2016.117 (2016).

13. Mcmurdie, P. J. \& Holmes, S. phyloseq: an R package for reproducible interactive analysis and graphics of microbiome census data. PloS ONE 8, e61217 (2013).

Open Access This article is licensed under a Creative Commons Attribution 4.0 International License, which permits use, sharing, adaptation, distribution and reproduction in any medium or format, as long as you give appropriate credit to the original author(s) and the source, provide a link to the Creative Commons license, and indicate if changes were made. The images or other third party material in this article are included in the article's Creative Commons license, unless indicated otherwise in a credit line to the material. If material is not included in the article's Creative Commons license and your intended use is not permitted by statutory regulation or exceeds the permitted use, you will need to obtain permission directly from the copyright holder. To view a copy of this license, visit http://creativecommons. org/licenses/by/4.0/.

(c) The Author(s) 2018 